Original Article

\title{
Effects of a functional foot orthosis on the knee angle in the sagittal plane of college students in their 20s with flatfoot
}

\author{
KwangYong Park, MS ${ }^{1)}$, KyoChul Seo, $\mathrm{PhD}^{2)^{*}}$ \\ 1) Department of Rehabilitation Technology, Korea Nazarene University, Republic of Korea \\ 2) Department of Physical Therapy, Korea Nazarene University: 456 Ssangyong-dong, Seobuk-gu, \\ Cheonan, Chungnam 331-718, Republic of Korea
}

\begin{abstract}
Purpose] The purpose of this study was to examine the effects of a functional foot orthosis on the knee angle in the sagittal plane of college students in their 20s who had flatfoot. [Subjects and Methods] The subjects of this study were 20 college students diagnosed as having flatfoot. The variations of their knee angle (Q-angle) in the sagittal plane during the stance phase were measured using the VICON Motion System (Vicon, Hansung, Korea) before and while wearing a foot orthosis. The experimental data were analyzed using SPSS 12.0 for Windows. [Results] The Q-angle in the test group during the stance phase showed statistically significant declines on the right and left sides while wearing the foot orthosis during the gait-phases of loading response and midstance. During initial contact, terminal stance, and preswing, the Q-angle also decreased on the right and left sides after wearing the foot orthosis, but the changes were not statistically significant. [Conclusion] The college students with flatfoot exhibited declines in the Q-angle in the sagittal plane while wearing a foot orthosis. In this regard, the application of active gait training using orthotic shoes for long hours is likely to help individuals with flatfoot to achieve normal gait. Key words: Flat feet, VICON, Q-angle
\end{abstract}

(This article was submitted Oct. 20, 2014, and was accepted Dec. 16, 2014)

\section{INTRODUCTION}

Flatfeet is a chronic or abnormal lowering of the medial longitudinal arch of the foot, which leads to the overstretching of the plantar fascia, spring ligament, and posterior tibialis tendon ${ }^{1)}$. As this causes weakening in the plantar ligament and fascia, the ability to bear or distribute the human body weight is reduced. This generates excessive compensatory actions which can also cause the overuse syndrome and foot imbalance ${ }^{2)}$. Human feet and ankles perform a primary function in postural control and balance maintenance ${ }^{3)}$. Therefore, when individuals with flatfoot carry out repeated physical activities, pain can occur in the lower extremities due to their acute or chronic overuse. These circumstances, they can experience spasms and early muscular fatigue in the lower extremities, heel pain, and chronic fatigue ${ }^{4}$.

Pratt $^{5)}$ conducted a study of functional foot orthoses to resolve foot deformities and reported that the tibial transition to internal rotation is reduced by preventing or correcting the deformities. Cornwall and $\mathrm{McPoil}^{6)}$ and Song ${ }^{7)}$ noted that foot orthotics reduce not only foot pronation, but also

*Corresponding author. KyoChul Seo (E-mail: blueskyskc@ nate.com)

(C2015 The Society of Physical Therapy Science. Published by IPEC Inc. This is an open-access article distributed under the terms of the Creative Commons Attribution Non-Commercial No Derivatives (by-ncnd) License $<$ http://creativecommons.org/licenses/by-nc-nd/3.0/> . tibial internal rotation. Nigg et al. ${ }^{8)}$ reported that proper shoe insoles and orthoses can reduce muscle activities, provide a sense of comfort, and increase motor skills ${ }^{9}$. In addition, Park and Park ${ }^{10)}$ conducted a study of changes in the ankle joint angle of individuals with flatfoot in the sagittal plane after wearing a functional foot orthosis.

The studies cited above investigated gait function when using ankle foot orthoses. However, only a small number of studies have dealt with the knee which serves a pivotal role in gait. This study investigated the effects on college students in their 20s with flatfoot of wearing a functional foot orthosis to identify its effects on their Q-angle in the sagittal plane using the VICON Motion System.

\section{SUBJECTS AND METHODS}

\section{Subjects}

This study was conducted from April 20 to April 30, 2014, with 20 undergraduates of K University, Chungcheongnamdo, as subjects. The subjects had no history of musculoskeletal system disease and were diagnosed as having flat feet, determined as a calcaenal pitch angle that was less than $15^{\circ}$ in radiological measurements. After the subjects were selected, they received an explanation of the study and gave their consent to participation. The study was approved by the Clinical Trial Review Committee of Korea Nazarene University, after reviewing it according to the ethical principles of the Declaration of Helsinki. The general characteristics of the subjects who took part in the study are summarized 
in Table 1.

\section{Methods}

In this study, the functional foot orthosis worn by each subject was customized by patterning to fit the subject's foot shape. The orthosis was made of thermplastics and combined a high-density rebounding elastic pad, a cupsole for the support of plantar arches, a low-elasticity pad for the absorption of heel impacts, and ethylenevinylacetate (EVA). The foot orthoses produced for the subjects had an the insole that was basically designed to keep the heel bone perpendicular to the ground. In addition, each subject's feet were measured and evaluated for weight bearing while the foot region was rearranged to prevent foot pronation and exclude excessive movements of the entire foot by enabling the subtalar joint to maintain a neutral position. After this process, the insole was produced by casting the plantar surface and following the procedures by which the company Alfoots (Korea) was requested to produce it. First, a tester measured and evaluated the feet of each subject and modeled the shape of each foot using Pedilen Foam. A positive plaster model was then prepared and underwent plaster modification (checking compressed or sensitive regions in the foot), and then a shell was produced by modeling with thermoplastic plastics. After that, posting and polishing (trimming and array adjustment) were performed. Finally, the foot orthosis was completed by covering ${ }^{11,12)}$.

This study used six MX-F40 cameras (VICON, Hansung, Korea) and two force plates, OR6-7, captured by for data collection, and Nexus software for data processing. The MX cameras reorganize two-dimensional images of the optical markers each camera into three-dimensional images. In doing so, they calculate not only the location data of each marker, but also the physical values of each body segment. The cameras are capable of measuring up to 2,000 fps. The two force plate indicate the ground reaction force of each foot as a vector. The Ultranet system performed the role of incorporating kinetic data derived from the cameras and kinematic data derived from the force plates into the same frame.

In terms of research procedures, the plug-in gait model based on the Newington-Helen Hayes gait model was used to calculate knee joint angles in the sagittal plane. These angles were measured using the VICON Motion System, which is a three-dimensional motion capture system, and based on the three-dimensional spatial coordinates of each marker point measured. Each segment's regional coordinate system was calculated and the Euler angles derived. One gait cycle was defined as starting from the heel strike, and a gait cycle out of the gaits of each of the subjects was selected and used for time normalization.

This study performed gait analysis with high-performance 3D cameras. The subjects were asked to walk on a predesigned walkway and their motion was captured using the VICON Motion System (Vicon, Hansung, Korea) to measure changes in their knee angle in the sagittal plane before and while wearing a customized functional foot orthosis. Markers were placed over the anterior superior iliac spine (ASIS) and the center of the knee of the subjects to measure their Q-angle during the stance phase. In addition, the knee angle
Table 1. General characteristics of the subjects

\begin{tabular}{lc}
\hline & Subjects $(\mathrm{n}=20)$ \\
\hline Gender $(\mathrm{M} / \mathrm{F})$ & $12 / 8$ \\
Age $(\mathrm{yrs})$ & $21.9 \pm 0.7$ \\
Height $(\mathrm{cm})$ & $171.4 \pm 8.3$ \\
Weight $(\mathrm{kg})$ & $67.8 \pm 9.8$ \\
Calcaneal pitch angle $\left({ }^{\circ}\right)$ & $14.2 \pm 0.5$ \\
\hline
\end{tabular}

Values are mean \pm standard deviation

Table 2. A comparison of the gait measures of before and while wearing the foot orthosis

\begin{tabular}{lccc}
\hline & & Before & Wearing \\
\hline Initial contact $\left({ }^{\circ}\right)$ & $\mathrm{R}$ & $-1.2 \pm 1.4$ & $-1.1 \pm 0.9$ \\
& $\mathrm{~L}$ & $-0.1 \pm 0.8$ & $0.0 \pm 0.7$ \\
Loading response $\left(^{\circ}\right)$ & $\mathrm{R}^{*}$ & $-0.4 \pm 1.5$ & $-0.1 \pm 1.5$ \\
& $\mathrm{~L}^{*}$ & $1.2 \pm 1.1$ & $0.7 \pm 0.9$ \\
Midstance $\left({ }^{\circ}\right)$ & $\mathrm{R}^{*}$ & $0.8 \pm 1.8$ & $0.5 \pm 1.6$ \\
& $\mathrm{~L}^{*}$ & $2.0 \pm 1.3$ & $1.86 \pm 1.2$ \\
Terminal stance $\left({ }^{\circ}\right)$ & $\mathrm{R}$ & $-0.8 \pm 1.1$ & $-0.2 \pm 1.0$ \\
& $\mathrm{~L}$ & $1.2 \pm 0.8$ & $1.1 \pm 0.7$ \\
Preswing $\left({ }^{\circ}\right)$ & $\mathrm{R}$ & $0.1 \pm 2.0$ & $0.0 \pm 1.8$ \\
& $\mathrm{~L}$ & $1.4 \pm 1.3$ & $1.3 \pm 1.1$ \\
\hline
\end{tabular}

Values are mean \pm SD; R: Right side; L: Left side. * Significant difference from before orthosis. $<0.05$.

difference between the left and right sides was calculated by quantifying dynamic variations. All subjects were instructed to repeat preliminary motions ten times to help them become familiar with the main experiment. The mean data of three trials was used in the statistical analysis. SPSS 12.0 for Windows was used for data analysis. The paired t-test was performed to analyze changes before and while wearing the foot orthosis. Statistical significance was accepted for values of $\mathrm{p}<0.05$.

\section{RESULTS}

The test group showed statistically significant declines in the Q-angle on the right and left sides while wearing the foot orthosis during the loading response and midstance in the stance phase $(\mathrm{p}<0.05)$. The Q-angle also decreased on the right and left sides of the subjects during initial contact, terminal stance, and pre-swing when foot orthoses were worn, but the decreases were not statistically significant ( $\mathrm{p}$ $>0.05$ ) (Table 2).

\section{DISCUSSION}

This study hypothesized that wearing a functional foot orthosis would have large effects on the knee of individuals with flatfoot in their gait cycle. Therefore, this study examined the changes during the stance phase in the Q-angle of college students in their 20s with flatfoot before and while wearing functional foot orthoses. The subjects exhibited statistically significant declines in the Q-angle on the right and 
left sides while wearing the foot orthosis during the loading response and at midstance in the stance phase. The subjects also showed decreases in the Q-angle on the right and left sides during initial contact, terminal stance, and pre-swing, but the desreases were not statistically significant. This suggests that wearing a functional foot orthosis tailored to each individual's foot shape relaxed tension in the plantar muscle and fascia of the subjects with flatfoot during the stance phase and elevated the talus joint, which led to inversion of the ankle joint. This may have decreased the Q-angle between the tibia and the femur.

Functional foot orthoses support the height of foot arches, preventing the collapse of foot arches during dynamic motions ${ }^{13)}$, helping to restore each joint's abnormal displacement to its normal position. Park and Park found that wearing a functional foot orthosis elicited statistically significant changes in the ankle joint angle of subjects through a mechanical shift of the joint below the talus in the sagittal plane. Wu Kent ${ }^{14)}$ reported that foot orthoses were used to align the foot region, prevent and correct foot deformities, and improve the foot region's functions. In addition, when individuals with flatfoot develop ankle and foot disorders, they can change their gait pattern using a functional foot orthosis, which influences the other joints of the lower extremities ${ }^{15)}$. Foot orthoses also play a role in dispersing plantar impacts evenly by performing functions which either could not be performed by the foot or became deficient in each gait phase $^{16)}$. In these previous studies, various patients showed improvement of gait parameters of their low extremities and foot region while wearing foot orthoses similar to the results of the present study in which changes were elicited in the Q-angle of the knee by foot orthosis.

Largest limitations of this study were the small number of subjects and the sample diversity which was limited to young individuals in their 20s and 30s. Future studies should address these limitations and investigated the movement changes of the entire lower extremities of subjects by including examination of the hip joint while subjects are wearing a functional foot orthosis.

\section{ACKNOWLEDGEMENT}

This Research was supported by the Korean Nazarene University Research Grants 2015.

\section{REFERENCES}

1) Houglum PA, Bertoti DB: Brunnstrom' Clinical kinesiology, 6th ed. F.A. Davis, 2012.

2) Neumann DA: Kinesiology of the musculoskeletal system, 2nd ed. Elsevier Korea, 2011.

3) Shumway-Cook A, Woollacott MH: Motor control, 2nd ed. Lippincott Williams \& Wilkins, 2001.

4) Moon JS, Bae WH, Seo JK, et al.: Clinical results of the subtalar arthroereisis for the flat foot. J Korean Foot Ankle Soc, 2008, 12: 117-121.

5) Pratt DJ: A critical review of the literature on foot orthoses. J Am Podiatr Med Assoc, 2000, 90: 339-341. [Medline] [CrossRef]

6) Cornwall MW, McPoil TG: Relative movement of the navicular bone during normal walking. Foot Ankle Int, 1999, 20: 507-512. [Medline] [CrossRef]

7) Song JH: The Kinematic comparative study about effects of foot orthotics. Korean J Sport Sci, 2008, 19: 11-21.

8) Nigg BM, Nurse MA, Stefanyshyn DJ: Shoe inserts and orthotics for sport and physical activities. Med Sci Sports Exerc, 1999, 31: S421-S428. [Medline] [CrossRef]

9) Hannaford DR: Soft orthoses for athletes. J Am Podiatr Med Assoc, 1986, 76: 566-569. [Medline] [CrossRef]

10) Park KY, Park SH: Study on change of the flatfoot's ankle angle in sagittal plane before and after wearing FFO. J Biomed Engineering Res, 2010, 31: $67-73$.

11) Takata $Y$, Matsuoka S, Okumura N, et al.: Standing balance on the ground -the influence of flatfeet and insoles. J Phys Ther Sci, 2013, 25: 15191521. [Medline] [CrossRef]

12) Lee $Y$, Her JG, Choi Y, et al.: Effects of ankle-foot orthosis on lower limb muscle activities and static balance of stroke patients author's names. J Phys Ther Sci, 2014, 26: 179-182. [Medline] [CrossRef]

13) Franco AH: Pes cavus and pes planus. Analyses and treatment. Phys Ther, 1987, 67: 688-694. [Medline]

14) Wu KK: Foot Orthoses, Principles and Clinical Applications. Baltimore: Williams and Wilkins, 1990, p 97.

15) Magee DJ: Orthopedic Physical Assessment, 3rd ed. Philadelphia: WB. Saunders, 1997.

16) Hertel J, Sloss BR, Earl JE: Effect of foot orthotics on quadriceps and gluteus medius electromyographic activity during selected exercises. Arch Phys Med Rehabil, 2005, 86: 26-30. [Medline] [CrossRef] 